\title{
Martian atmospheric particulate spectral end-members recovery from PFS and IRIS data
}

\author{
Mario D’Amore $^{\mathrm{a}, *}$, Alessandro Maturilli ${ }^{\mathrm{a}}$, Angelo Zinzi ${ }^{\mathrm{b}, \mathrm{c}}$, Ernesto Palomba ${ }^{\mathrm{d}}$, Jörn Helbert ${ }^{\mathrm{a}}$ \\ ${ }^{a}$ DLR, Institute of Planetary Research, Rutherfordstraße 2, 12489 Berlin, Germany \\ b INAF-OAR, Via Frascati 33, 00040 Monte Porzio Catone (RM), Italy \\ 'ASDC, Via Galileo Galilei, 00044 Frascati (RM), Italy \\ d INAF-IAPS, Via del Fosso del Cavaliere 100, 00133 Roma, Italy
}

\section{A R T I C L E I N F O}

\section{Article history:}

Received 6 August 2012

Revised 14 June 2013

Accepted 5 August 2013

Available online 14 August 2013

\section{Keywords:}

Mars

Mars, atmosphere

Spectroscopy

\begin{abstract}
A B S T R A C T
We present an application of a multivariate analyses technique on data returned by the Planetary Fourier Spectrometer (PFS) instrument on board the ESA's Mars Express (MEX) spacecraft in order to separate the atmospheric contribution from the observed radiation. We observe that Thermal/Far Infrared spectra returned from Mars, covering almost a whole martian year, can be represented by a linear model using a limited set of end-member spectra. We identify the end-members as the suspended mineral dust and water ice clouds, but no surface signature was found. We improve previous studies performed with data from the Thermal Emission Spectrometer (TES) thanks to the higher spectral resolution of PFS. This allows for distinguishing narrow gaseous bands present in the martian atmosphere. Furthermore, the comparison of results from PFS and TES with data collected in 1971 by the Mariner 9 Infrared Interferometer Spectrometer (IRIS) shows an atmospheric dust component with similar spectral behavior. This might indicate homogeneity of the dust source regions over a time period of more than 30 years.
\end{abstract}

(c) 2013 Elsevier Inc. All rights reserved.

\section{Abstract}

In this work we present the first application of multivariate analyses techniques on data from the Planetary Fourier Spectrometer (PFS) instrument on board the ESA Mars Express (MEX) spacecraft in order to identify the atmospheric contribution to the observed radiation. The analyzed dataset covers the mid-infrared spectral range from 300 to $1300 \mathrm{~cm}^{-1}$, a region widely used to characterize key materials in planetary studies, such as minerals and gaseous species.

The data span from the end of the martian year (MY) 26 to the end of MY27, roughly from end 2004 to mid 2006 (see Clancy et al., 2000). A large regional dust storm in the middle northern winter of MY27 forced us to exclude this season, as our approximation is not valid in heavily dusty conditions. However, the resulting dataset covers about a whole martian year, a time interval adequate to monitor all the distinctive features of the martian atmosphere.

Our major focus was the characterization of atmospheric components. We only analyzed data collected over surfaces with high emissivity in the wavelength regions studied, selected following previous TES results (Bandfield et al., 2000). Without this constraint, the sur-

\footnotetext{
* Corresponding author.

E-mail addresses: Mario.Damore@dlr.de (M. D’Amore), Alessandro.Maturilli@ dlr.de (A. Maturilli), zinzi@asdc.asi.it (A. Zinzi), ernesto.palomba@ifsi-roma.inaf.it (E. Palomba), Joern.Helbert@dlr.de (J. Helbert).
}

face generally contributes significantly to the total observed radiation. However in high emissivity areas the surface contribution is negligible, while it has been demonstrated that surface components are consistently present at other locations (Maturilli et al., 2009).

This first application of multivariate techniques on the PFS spectra confirms that they can be fully represented by a linear model using only a limited set of end-member spectra. The latter are identified by a target transformation technique, and represent the major components of the martian atmosphere, i.e. the suspended mineral dust and water ice clouds, with the narrow gaseous band present in the martian atmosphere clearly resolved by PFS.

With the same technique used for the PFS, we also analyzed a set of data collected in 1971 by the Mariner 9 Infrared Interferometer Spectrometer (IRIS), extracting the components that contribute to the observed radiation.

The comparison between our results for PFS and IRIS and those for TES (Bandfield et al., 2000) shows that they are in good consistent with one another, allowing for speculation on the homogeneity and temporal stability of the dust source regions.

\section{Introduction}

The martian atmosphere is a complex system where aerosols act as key components. Suspended dust and water ice clouds strongly influence the atmospheric thermal field, which in turn affects water 
ice equilibrium, wind strength, and surface particulate uplift. Several phenomena, such as dust storms and seasonal water ice cloud systems (e.g. the polar hood, the equatorial cloud belt), strongly influence the surface and atmosphere temperature environment.

These processes have been widely studied, for example, by the Mars Orbiter Camera (e.g. Edgett and Malin, 2000; Cantor, 2002), and the Thermal Emission Spectrometer (TES) (Smith et al., 2001). The scientific payload of the Mars Express spacecraft is still monitoring Mars, extending our knowledge of the surface, the geological structures and the climate.

The atmospheric particulates in the martian atmosphere show extreme geographical and temporal variability, both in optical depths and in particle sizes (e.g. Benson et al., 2003; Clancy, 2003; Wolff, 2003; Zinzi et al., 2010), producing a typical dust opacity at $9 \mu \mathrm{m}$ ranging from less than 0.1 in clear conditions up to more than 2 during dust storms (Wolff, 2003). Since atmospheric dust is a strong absorber in the thermal infrared, it has a large impact on radiative heating and, consequently, on the thermal and dynamical state of the atmosphere.

The water ice clouds show even more variability, from tiny hazes to vast polar hoods (Smith, 2004), albeit total water ice opacity is generally much lower than total dust opacity. Water ice clouds influence the martian climate through their role in the water cycle. Ice clouds are also present at tropical latitudes, mainly during martian northern summer, and their structures are strongly related to topographic features, such as the domes of Tharsis, Olympus and Elysium Mons. Above these volcanoes, ice clouds are also sporadically observed in other seasons (Zasova et al., 2005).

In this work, we analyse the data from PFS (Formisano et al., 2005 ) to extract spectral properties of the atmospheric particulates via multivariate analyses techniques. The foundations of these techniques were successfully proofed on the TES data by Bandfield et al. (2000). As an initial guess for the atmospheric particulates we use spectral properties based on the canonical atmospheric shapes obtained from the TES analysis, publicly accessible at the TES website from Arizona State University.

In addition, the data collected by IRIS during the early '70s, almost 30 years before the TES and PFS investigations, have been selected and analyzed by using the same techniques.

To cover a large atmospheric variability, PFS data are selected to span a period showing large variation of particulates abundance, only excluding atmospheric conditions that are very severe, as with the large regional dust storm during the MY27 at Ls $\sim 300^{\circ}$. These techniques based on linear models are demonstrated to be able to retrieve the composition of laboratory samples (Smith et al., 1985) and to separate the principal varying components within a large spectral dataset in the thermal infrared (Bandfield et al., 2000).

Smith et al. (2000a, 2000b) have shown that infrared observations on warm surfaces could be closely reproduced by a linear model of appropriate atmospheric and surface end-members. The atmospheric aerosols scattering is negligible for low opacity and small phase angle observations, where for limb conditions even a small amount of atmospheric particulate could have a strong influence.

This allows us to directly model the transmission spectra of suspended mineral dust, as seen by the different instruments, and to address whether the dust component of a suspended particulate shows a significant temporal variation.

\section{Instrument and dataset}

The Mars Express spacecraft is in an elliptical orbit (250 km × 10,000 km) around Mars (Hechler and Yáñez, 2003).
Its scientific operations began in January 2004 or mid martian year 26 (Clancy et al., 2000) and have continuously monitored Mars up until today. PFS is an infrared double-pendulum interferometer split into two channels, the short wavelength and the long wavelength one (SWC and LWC, respectively). The LWC acquires data in a spectral range dominated by both reflected solar radiation and thermal radiation emitted from planetary surfaces, roughly between $270 \mathrm{~cm}^{-1}$ and $1800 \mathrm{~cm}^{-1}$, with a sampling of about $1 \mathrm{~cm}^{-1}$ and an apodized spectral resolution of around $2 \mathrm{~cm}^{-1}$. The field of view of the LWC has a diameter of $2.8^{\circ}$, which is equivalent to an area of about $12.5 \mathrm{~km}$ diameter on the surface at pericenter. Details on the instrument and its radiometric performances can be found in Formisano et al. (2005) and Giuranna et al. (2005). Thermal profile and atmospheric particulates opacities are systematically retrieved from the LWC channel from the PFS team via a lineby-line radiative transfer code coupled with a Bayesian approach (Grassi et al., 2005).

To fit data quality conditions (Smith et al., 2000a), data are filtered by geographical and temporal criteria in order to emphasize atmospheric variations and to observe only warm daily bright surfaces $\left(T_{\text {surf }} \geqslant 260 \mathrm{~K}\right)$. The spectra are converted to apparent emissivity by dividing out a Planck curve of the highest brightness temperature within a band of $100 \mathrm{~cm}^{-1}$ width in each spectrum, that was found around $1275 \mathrm{~cm}^{-1}$ in all cases. Selected observations span a narrow local time range around noon that automatically excludes night observations.

The initial PFS dataset used starts at the latest northern winter in MY26 (solar longitude Ls 330 ${ }^{\circ}$ ) and spans the whole martian year until the Global Dust Storm observed during MY27 at Ls $\sim 300^{\circ}$, including data from orbit 10 to orbit 1918 of the mission nominal phase. The storm was so intense that data from this period had to be excluded. During such severe atmospheric conditions the multiple scattering in the atmosphere becomes important. The approach presented here is not applicable, because radiation transport through a strong opaque atmosphere cannot be linearly approximated. Neither the particulate composition nor the particle size distribution variation can be distinguished in the data using a linear mixing approach, however in this period those properties are likely to vary significantly.

The data in this study were selected to emphasize atmospheric components. This was done by focusing on periods of relatively high dust opacity (e.g. Smith et al., 2000b) as well as using data collected over surfaces with high emissivity in the wavelength regions studied. These surfaces were selected based on previous TES results (Bandfield et al., 2000). While surface components are either not present or subdued in these locations and wavelength regions, it has been demonstrated that surface components are consistently present at other locations (Maturilli et al., 2009). Therefore thanks to the selection criteria here used, the surface contribution to the observed radiation can be considered negligible.

The process bins about 200,000 spectra on the basis of retrieved atmospheric dust ( $\left.\tau_{\text {dust }}\right)$ and water ice cloud column opacity $\left(\tau_{\text {ice }}\right)$ (measured in terms of integrated optical thickness at $1100 \mathrm{~cm}^{-1}$ and $850 \mathrm{~cm}^{-1}$ respectively, see Grassi et al., 2005) into intervals of $0.1 \tau$ (Fig. 1 ). The average spectrum of each bin thus characterizes an atmospheric scenario for a given interval of $\tau_{\text {dust }}$ and $\tau_{\text {ice. }}$. The maximum total opacity for both atmospheric components was limited to 0.5 to avoid that nonlinear scattering effects become prevalent (Smith et al., 2000a). This automatically eliminates some extreme local conditions present in a few spectra. The subset of averaged spectra is analyzed, allowing us to identify martian atmospheric varying components and separate them into pure single components. In addition, we analyzed in depth the data from orbit 37 acquired at the end of the Northern winter. This orbit is a nearly longitude-constant south to north section of the planet, performed during the middle northern winter $\left(\mathrm{Ls} \sim 337^{\circ}\right.$ ). The spacecraft flew 


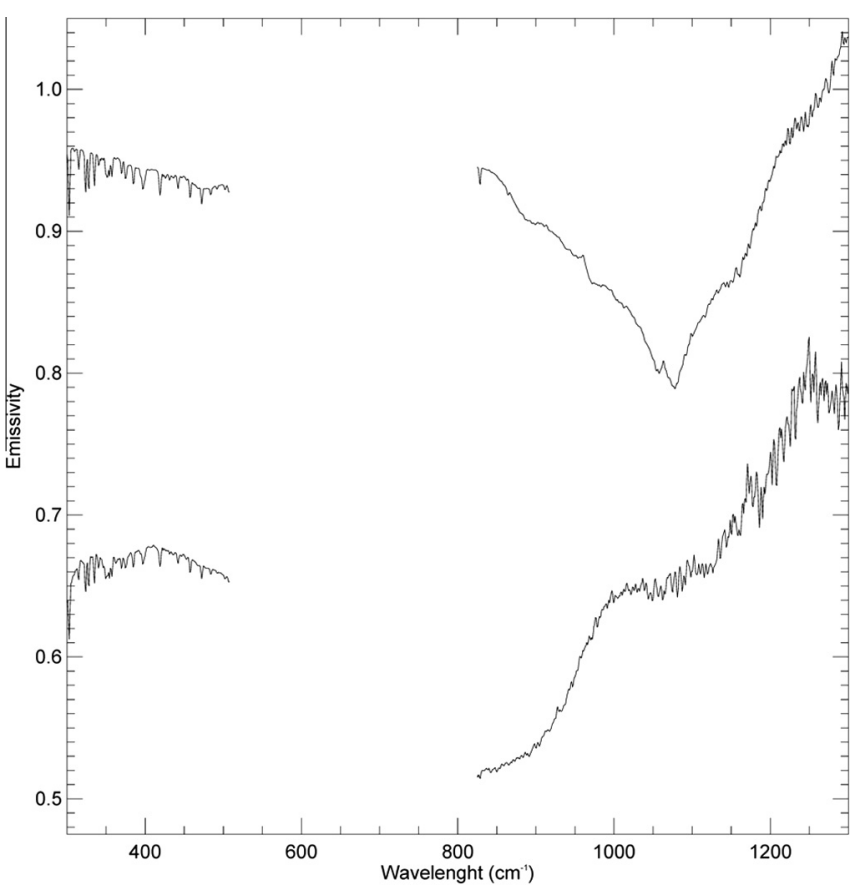

Fig. 1. Above spectrum represents a scenario with dust total opacity at $1100 \mathrm{~cm}^{-1}$ between $0.2-0.3$ and water ice total opacity at $850 \mathrm{~cm}^{-1}$ less than 0.1 . Bottom spectrum (shifted down by 0.2 in emissivity) represents dust opacity less than 0.1 and water ice opacity between $0.3 / 0.4$, both as measured by PFS.

directly over the Olympus Mons caldera (Fig. 2 and Table 1), where the altimetry computed by Mars Orbiter Laser Altimeter (MOLA) ranges from -3 to $21 \mathrm{~km}$. Due to the considerable variation in altimetry, we expect to see a large atmospheric variability at this time of the year. The field of view ranges from $20 \mathrm{~km}$ at pericenter (around at the equator) to $60 \mathrm{~km}$ at both edges of the observation session (Fig. 2). Local time spans $20 \mathrm{~min}$ around $1 \mathrm{PM}$ and consequently the incidence angle shows little variation from $20^{\circ}$ to $40^{\circ}$, while the emission angle ranges from $10^{\circ}$ to $30^{\circ}$.

\section{Methodology}

In the present work a combination of R-mode factor analysis and target transformation (Malinowski, 2002) is applied to retrieve and characterize the number and the spectral shape of the varying components present in the PFS dataset. Reliability of linear modelbased techniques is well established in remote sensing and in laboratory mineral mixtures analysis (e.g. Gillespie, 1992; Ramsey et al., 1993; Thomson and Salisbury, 1993; Ramsey and Christensen, 1998; Bandfield et al., 2000; Smith et al., 2000a).

The implemented steps are:

- Selection of the dataset to be analyzed, assuming that any variability in it could only be ascribed to a varying combination of spectral end-members.

- Calculation of the covariance matrix and extraction of the eigenvector and eigenvalues, determining the number of independent vectors (principal components) needed to reconstruct the original measured spectra within the noise level. This number also defines the dimensionality of the data set.

- Identification of spectral end-members by the least square fitting of representative trial spectra (usually from a spectral library) to the principal components. This step, due to the limits of spectral libraries, does not assure an optimal solution. However, if the differences between true spectral end-members and

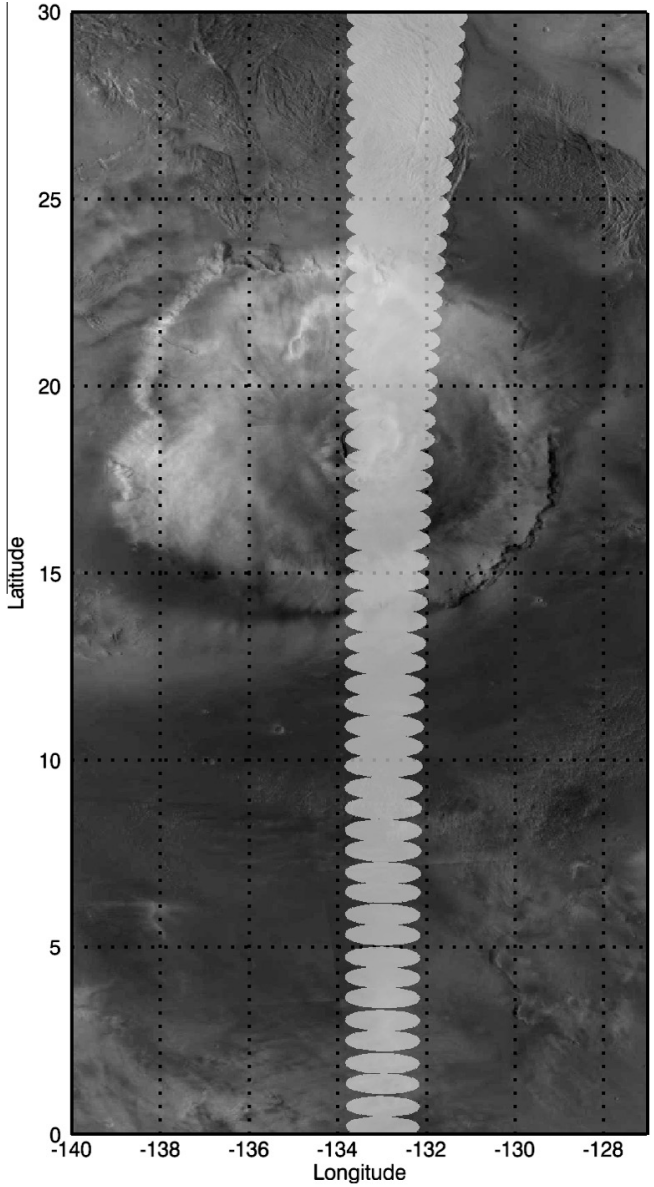

Fig. 2. PFS instantaneous field of view during orbit 37. The orbit passes directly over the Olympus Mons caldera.

Table 1

Observational parameter for the analyzed orbit.

\begin{tabular}{lll}
\hline & Orbit 37 & \\
\cline { 2 - 3 } & Min & Max \\
\hline Solar longitude $\left(^{\circ}\right)$ & 337.11 & 337.11 \\
MOLA altimetry $(\mathrm{km})$ & -2.2 & 21.1 \\
Incidence angle $\left(^{\circ}\right)$ & 28 & 50 \\
Emission angle $\left(^{\circ}\right)$ & 19 & 41 \\
Phase angle $\left(^{\circ}\right)$ & 12 & 38 \\
Albedo & 0.13 & 0.31 \\
Local time & $13: 50$ & $14: 08$ \\
Spacecraft altitude $(\mathrm{km})$ & 273 & 735 \\
Major Ax. & 81 & 81 \\
Minor Ax. & 13 & 13 \\
\hline
\end{tabular}

trial spectra can be accounted for by a linear combination of principal components, then the least square fit of trial spectra will represent a plausible spectral end-member.

- Least square fitting of the dataset to selected end-members and visual inspection of residual errors as goodness-of-fit indicator.

\subsection{Factor analysis}

The factor analysis is designed to find a vectorial basis where the data covariance matrix is minimized. The solution to this optimization problem is the eigenvector base that diagonalizes the covariance matrix. The covariance matrix is essentially an array of covariance between couples of measured elements. It is the nat- 
ural generalization to higher dimensions for the concept of variance of a scalar random variable.

The number of independent variables needed to describe the dataset is generally less than the dimensionality of the covariance matrix, but the repetitive nature of the measurement process leads to a larger dataset. Therefore, this "true" dimensionality must be evaluated from the data by defining the number of principal components of the system.

For the sake of clarity, lets us suppose there is a true dimensionality of two. Any dataset could then be expressed as points lying on a bi-dimensional plane, regardless of how many instrumental bands of measurement we have. Points will be scattered by the noise introduced by the measurement process. Factor analysis can also be regarded as a compression algorithm, suited to eliminate inessential noise and to retain only the most important sources of information present in the data.

The eigenvalues of the covariance matrix are evaluated by ad hoc numerical codes and the data are decomposed in the space generated by the eigenvectors. Larger eigenvalues are associated with the larger part of the variance and then correspondent eigenvectors account for the larger part of the variance in the dataset. The smaller the eigenvalues, the more associated eigenvectors are featureless and contribute very little to the data reconstruction. Therefore, small eigenvalues are mainly associated with noise (and are also called secondary eigenvalues).

The individuation of the correct principal components is a critical task and involves expert user knowledge. Several methods have been developed to recognize the right number of principal components present in a dataset. In this work we used a combination of three methods: eigenvalues ratio (Bandfield et al., 2000), reconstruction error (Malinowski, 2002) and visual inspection of spectra.

Formally, spectra in the dataset are assembled in a matrix form such as

$\underline{\mathbf{D}}=\underline{\mathbf{R}} \cdot \underline{\mathbf{C}}$

where $\mathbf{D}$ is the matrix of the data, $\mathbf{R}$ the matrix of reconstructing vectors and $\underline{\mathbf{C}}$ the concentration coefficients matrix. The technique attempts to decompose $\mathbf{D}$ in two matrices (or in other words to diagonalize the $\underline{\mathbf{D}}$ matrix), with $\underline{\mathbf{R}}$ made up by the eigenvector of $\underline{\mathbf{D}}$.

\subsection{Determining the number of factors}

Evaluating the number of vectors needed to reconstruct the data within the experimental noise is a critical issue for converging to an accurate solution. Selecting a number of eigenvectors smaller than the original dimensionality is equivalent to assuming that the underlying independent variables composing the dataset are less than the number of measurements. Modeling data as a combination of a limited number of components has the advantage of exposing the data hidden structure, buried in redundant measurements, which can be more easily interpreted than in the original bigger dataset.

The first step to finding the number of principal components is the analysis of the eigenvalues ratio: in fact, the boundary between the $\mathbf{n}$ primary eigenvalues and secondary eigenvalues is normally indicated by an abrupt increase in the ratio value (Malinowski, 2002) that is flattened by a high level of noise.

The second step is the number of principal components evaluation. This is obtained by minimizing the total reconstruction error varying the number of principal components to reconstruct the data in a linear way. At this point first the most significant eigenvectors (larger eigenvalues) are used, follow by subsequent minor eigenvectors. When the residual error does not vary appreciably, we can be reasonably confident that the selected eigenvectors represent an adequate base to express the data.
Instrument performance parameters are used (i.e. embedded noise in the data, noise equivalent ratio) to check if the data reconstruction model error is negligible or if the model is still nonoptimal.

\subsection{Identification of spectral end-members}

When the eigenvectors, eigenvalues and $\mathbf{n}$ are estimated, then each single principal component vector should be assigned to a spectrum of an actual specimen.

This is accomplished by least square fitting a collection of significant trial spectra from a spectral library to the principal components.

This is also called target transformation and in algebraic notation could be regarded as a rotation of the $\mathbf{R}$ abstract matrix (see Eq. (1)) in a set of physically meaningful vectors, those coming from a spectral library of known materials (in our case gaseous species, minerals or water ice). This step is obviously necessary, because $\underline{\mathbf{R}}$ matrix vectors have no immediate physical interpretation and usually mix different spectral features from different constituents. The rotation is accomplished by projecting a set of test vectors from the spectral reference library into the vector space generated by the principal eigenvector. A projection close to its test vector implies that the original vector fits well into the space generated by the significant vector in $\underline{\mathbf{R}}$, as could be shown by a simple algebraic manipulation.

Recalling the bi-dimensional example in Section 4.1, a test vector that closely matches its projection has a small off-plane component (Fig. 3) that is negligible for the instrument. Obviously, off-plane components smaller than the detection level of the instrument (influenced by, e.g., calibration errors, resolving power, spectral resolution) cannot be detected. Therefore, instrument performance drives the choice of the spectral library and experience is essential to exclude samples without meaning for the current application. The $\underline{\mathbf{R}}$ matrix projects a set of test vectors $\underline{\mathbf{t}}_{n}$ as

$\underline{\mathbf{x}}_{\mathrm{b}}=\underline{\mathbf{R}} \cdot \underline{\mathbf{t}}_{\mathrm{n}}$

where $\underline{\mathbf{t}}_{\mathrm{n}}$ is a spectrum from the trial library, supposed to be a constituent of the sample, and $\underline{\mathbf{x}}_{\mathrm{b}}$ is the projection of the test spectrum in the space generated by $\underline{\mathbf{R}}$. If the distance $\left|\underline{\mathbf{x}}_{\mathrm{b}}-\underline{\mathbf{t}}_{\mathrm{n}}\right|$ is small enough, the original vector $\underline{\mathbf{x}}_{\mathrm{b}}$ belong to the space generated by $\underline{\mathbf{R}}$ and can be assembled in a vector set.

When $\left|\underline{\mathbf{x}}_{\mathrm{b}}-\mathbf{t}_{\mathrm{n}}\right|$ is not insignificant, the $\mathbf{x}_{\mathrm{b}}$ vector could represent an accurate reconstruction of the actual pure component found in $\underline{\mathbf{D}}$ (Bandfield et al., 2000), even if it does not fit into the space generated by $\underline{\mathbf{R}}$. That is especially useful if the pure component represented by $\underline{\mathbf{x}}_{\mathrm{b}}$ is not present in the test library, a common situation due to finite spectral libraries. That means all the possible samples

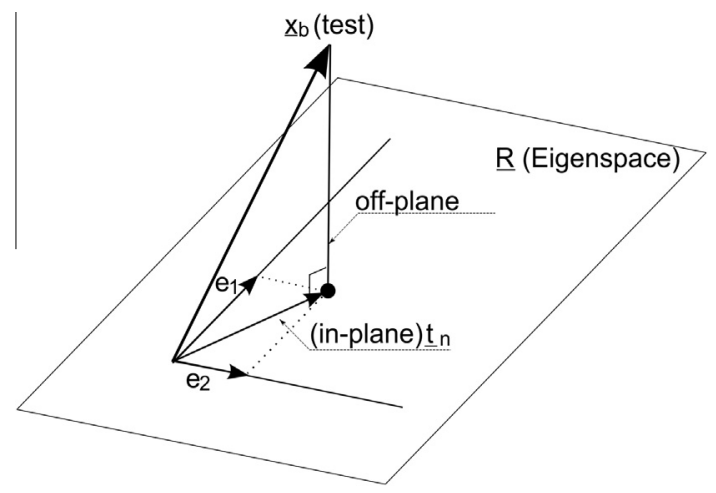

Fig. 3. Target transformation example in $2 \mathrm{D}$. Test vector $\mathbf{x}_{\mathrm{b}}$ from library is projected in the space generated by the principal components (eigenspace) in the vector $\mathbf{t}_{\mathrm{n}}$. Off-plane component measures the co-linearity of the test vector and its projection into the plane. 
variability cannot be included in a spectral library, because morphological, chemical and mineralogical variations could affect the spectra of a single material (e.g. Farmer, 1974; Hapke, 1981; Salisbury et al., 1987; Hamilton and Gemperline, 1990; Salisbury, 1991; Moersch and Christensen, 1995).

Because actual spectral features present in the observed dataset are combined to compose the projected $\underline{t}_{n}$, this procedure implicitly defines a method of building missing spectral components. Plausible end-members are chosen to build a vectorial base to linearly reconstruct the original dataset, substituting the abstract eigenvectors base. This process essentially gives a set of independent vectors with the same dimensionality of the space generated by $\underline{\mathbf{R}}$.

Trial library assembly and selection of projected vectors potentially introduce user bias in the analysis process. Some well known constituents of the martian atmosphere (Hanel et al., 1972; Smith et al., 2000b; Pearl et al., 2001) are expected to be found in the data, namely atmospheric particulates (dust, ice clouds) and atmospheric gases, mainly $\mathrm{CO}_{2}$ and $\mathrm{H}_{2} \mathrm{O}$ signatures.

The next section demonstrates is shown how we successfully separate contributions due to atmospheric constituents and how we clearly resolve the narrow atmospheric gas lines between particulate bands in the constituent spectra, thanks to the high spectral resolution achieved by the PFS instrument.

The atmospheric component abundances, which are simultaneously retrieved in Eq. (1) by the $\underline{\mathbf{C}}$ matrix, could be used in further applications to evaluate particulates temporal evolution and to support atmospheric and surface contribution separation in areas with significant surface contribution to the observed spectra, following Smith et al. (2000a, 2000b).

\section{Application}

\subsection{General PFS end-members recovery}

As discussed in the previous section, our aim is to identify a set of conceivable atmospheric components as initial guesses for the target transformation end-member recovery of the PFS data.
Previous studies based on TES data (Bandfield et al., 2000; Smith et al., 2000a) showed that, apart from the high opaque $15 \mu \mathrm{m} \mathrm{CO} 2$ band, the most prominent features in the thermal infrared are due to suspended mineral dust and to water ice clouds.

Although it is possible for $\mathrm{CO}_{2}$ ice clouds to be present in equatorial regions in some circumstances (Montmessin et al., 2006), its spectral signature is essentially overlapping the $15 \mu \mathrm{m} \mathrm{CO} \mathrm{CO}_{2}$ band that is outside of the analyzed range, so that they are negligible in this study.

The factor analysis abstract reproduction of the spectrum representing the opacity bins clearly shows that the principal components varying in the dataset are the dust and water ice cloud spectral shapes (Fig. 4), plus a small component linked to the unison variation of the dust and water ice. The eigenvalues absolute values decrease rapidly, but their ratio start to increase starting from the 4th value, indicating that the first 3 values are significant. The further eigenvector, are thus classified as secondary, representing mostly noise and showing no spectral features. The first 3 eigenvectors describe more than $99 \%$ of the variability of the data. Consistently with our initial selection, no surface signature is evident from the extracted eigenvectors.

To recover the PFS spectral end-members, we use the canonical atmospheric shapes obtained from the TES analysis as the trial spectral library, publicly available at the Arizona State University's TES website. Several authors (Bandfield et al., 2000; Smith et al., 2000a) demonstrate that these shapes closely fit every TES observation, occasionally using a locally derived end-member for components with low concentration levels (commonly the water ice clouds). We expect the same behavior for PFS data, even if the atmospheric gases could have a stronger effect due to the higher spectral resolution of this instrument. Target transformation projections (Fig. 5a and b) show that the test dust spectral shapes closely match their projection in the PFS eigenvector space, except for a difference in the broad band near $890 \mathrm{~cm}^{-1}$, that is not clearly discernible due to the presence of carbon dioxide or water vapor. This band is similar to a feature observed in transmission spectra calculated starting from IRIS derived optical constants by Hamilton

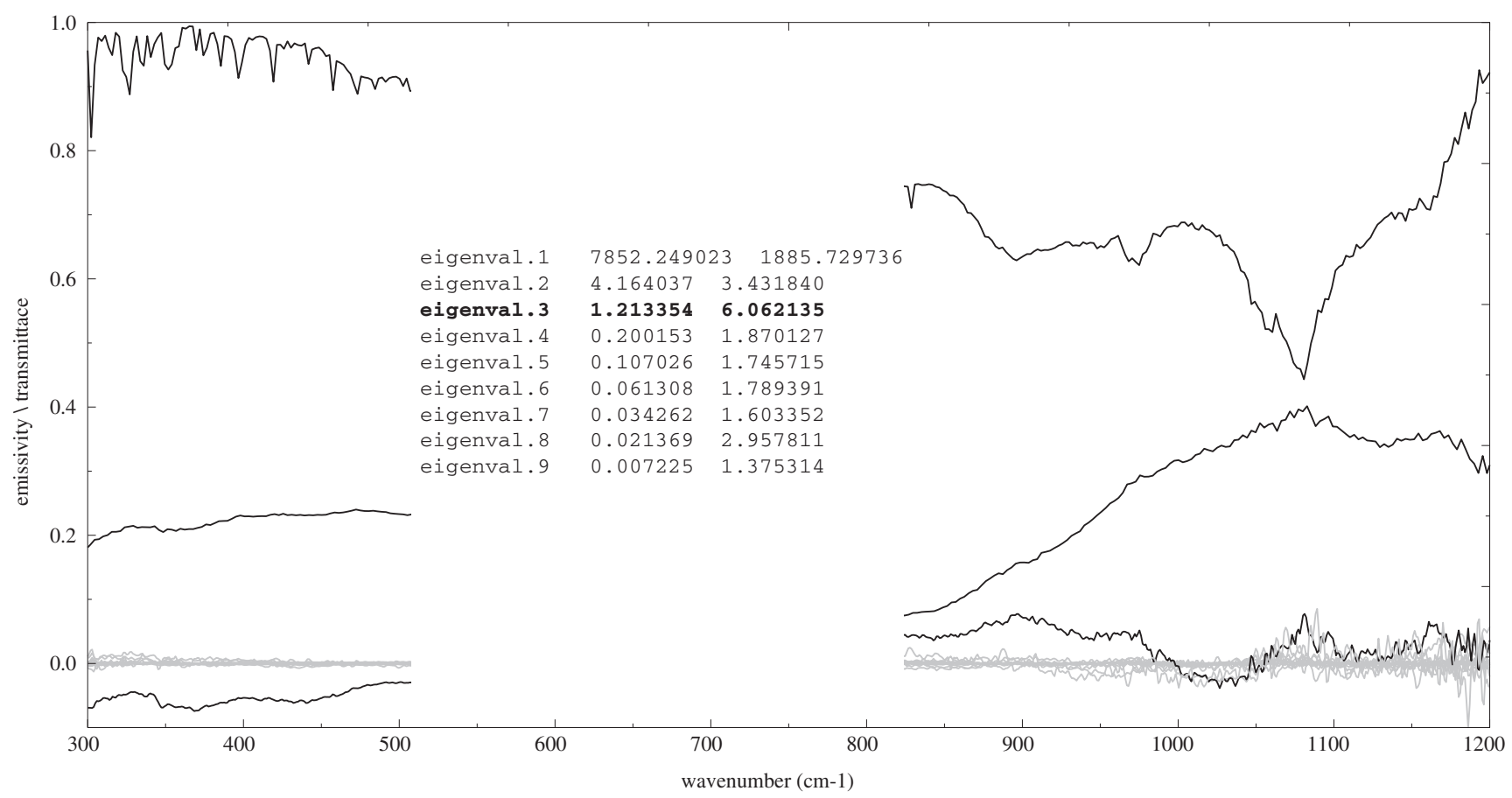

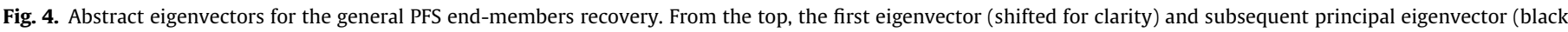
line) and the secondary eigenvector (gray line). The secondary are clearly related mostly to noise. The columns show the eigenvalues and their ratio. 
et al. (2005), where was observed that the feature is not due to silicate mineral, however, the nature of this band was not addressed.

Some major features in the TES shapes appear to be clearly due to atmospheric gases. In particular, the broad feature below $500 \mathrm{~cm}^{-1}$ comes from the convolution of several narrow gaseous bands, which can be observed in the $\mathrm{CO}_{2}$ and $\mathrm{H}_{2} \mathrm{O}$ synthetic spectra (Fig. 3a and b).

The influence of the strong $\mathrm{CO}_{2}$ bands above $900 \mathrm{~cm}^{-1}$ on the spectral shape retrieved is evident from Fig. $5 a-c$, especially the doublets centered around 960 and $1070 \mathrm{~cm}^{-1}$, while the doublets near $1250 \mathrm{~cm}^{-1}$ seem to leave the result almost unaffected, probably because the instrumental noise rapidly increases towards the high wavenumber region and could mask the actual features.

The two TES dust shapes, differing in the relative depth of gaseous $\mathrm{CO}_{2}$ hot bands (Smith et al., 2000a, 2000b) and the respective two PFS retrieved spectral shapes are shown in Fig. 5a and b. The two retrieved shapes are slightly different (Fig. 5c), but their ratio is quite smooth, indicating the same gaseous bands spectral contrast, with noise increasing towards high wavenumbers.

We recover other components whose gaseous lines could be clearly seen in the spectral shape representing ice clouds (Fig. 5d). This component exhibits more noise than for the dust, quickly increasing towards the higher wavenumbers, due to the general minor presence of ice in the atmosphere compared to dust. This was also a common condition also in the TES analysis and can be largely explained by the fact that target transformation and factor analysis are able to separate at best those components that show larger variability in the data. Components with small concentration and little variation in the data produce worse results because they contribute little to the data variance, thus being more difficult to separate from the noise.

The broad band centered at $1080 \mathrm{~cm}^{-1}$ (Fig. 5d) overlaps the broad dust absorption band. We cannot exclude a little mixing of spectral features in the retrieved component, resulting from subtle
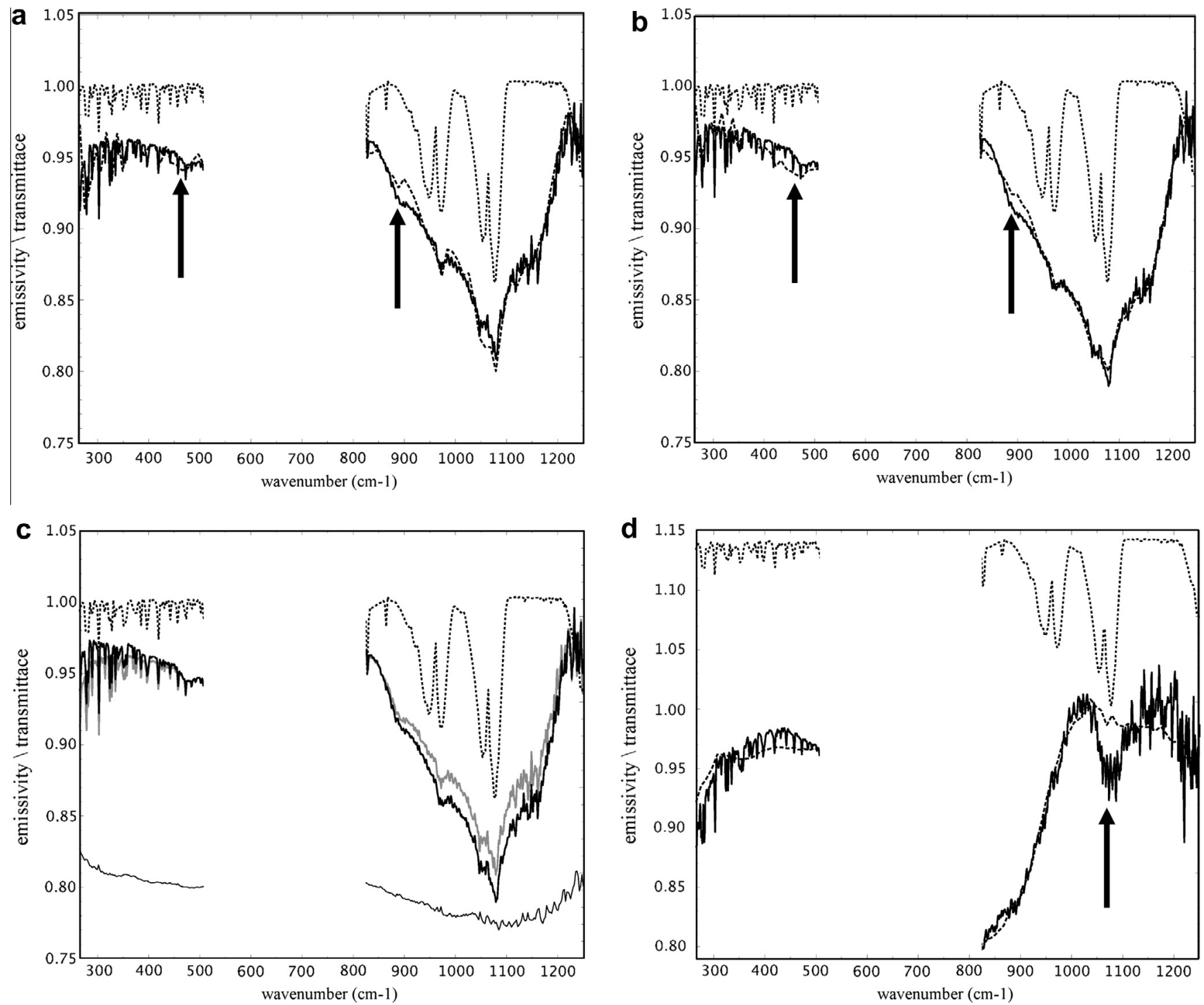

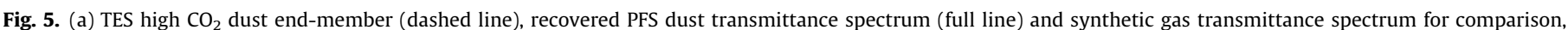

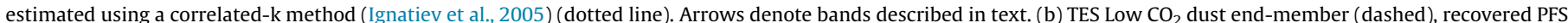

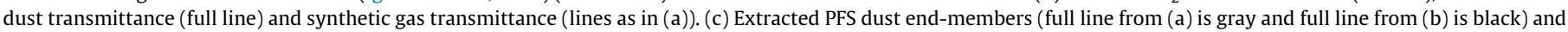

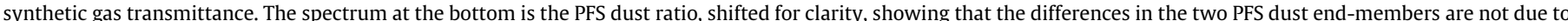

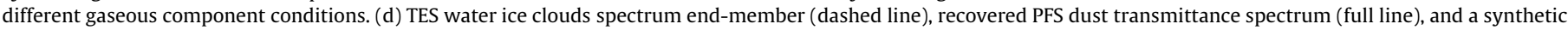
gas transmittance (dotted line). 
geographical correlation between the two components variation in the present dataset.

Overall, the target-transformed spectra and their initial guess largely coincide, indicating that the PFS end-members are close the initial TES end-member guess vectors.

\subsection{Application to PFS orbits}

We applied the described techniques to a single orbit data, in order to verify if the PFS data could be effectively described as a linear combination of the recovered general PFS atmospheric end-members. This application follows the original work by (Bandfield et al., 2000), using PFS data on Arsia Mons, acquired slightly early in the martian year. The area is chosen again to emphasize atmospheric components focusing on surfaces with high emissivity in the wavelength regions studied.

Since the PCA works at its best when the underlying components show great variation, we choosen a subset of the data where these variations are more likely to occur. In particular we selected an orbit acquired during the early scientific phase of the MEX spacecraft, namely the orbit 37 (Fig. 2 and Table 1). Even if no major atmospheric phenomena are expected in this season, like dust storms or the presence of massive water ice clouds, the dataset presents a considerable variation in the dust content (Fig. 6a, notice the great variation of the emissivity level around $1100 \mathrm{~cm}^{-1}$ ) due to the presence of the vast volcano (Zasova et al., 2005).
The PCA successfully extracts the independent eigenvectors of the dataset. Fig. 7 shows that, as in the previous application, eigenvalues decrease do not very rapidly, but the ratio ceases to decrease at the 5th value. We then choose to retain the first 4 eigenvectors and to assign the role of secondary to the following ones, that coherently do not show any signature in Fig. 7, representing mainly the noise embedded in the data.

The first eigenvector captures the main variation in the data, which is clearly due to the dust. The second eigenvector shows that variations of the broad dust absorption band relative to the first vector are present in the data. The third eigenvector is related to water ice present, as expected, at lower levels compared to dust and the forth shows a degree of correlation in dust and water ice variation.

The general PFS atmospheric end-members extracted in Section 5.1 are the initial guesses for the target transformation, to translate the abstract eigenvectors into a physically meaningful set of spectra.

The target transformation identifies the actual dust and water ice clouds spectral shape present in the dataset (Fig. 6b). The dust spectral shape closely matches our initial end-member guess, confirming our assumption that the PFS end-members obtained in Section 5.1 are consistent.

The water ice shape, on the other hand, is substantially different from its initial guess vector (Fig. 6b). The minor band depth for the major water ice absorption at $850 \mathrm{~cm}^{-1}$ could be due to known
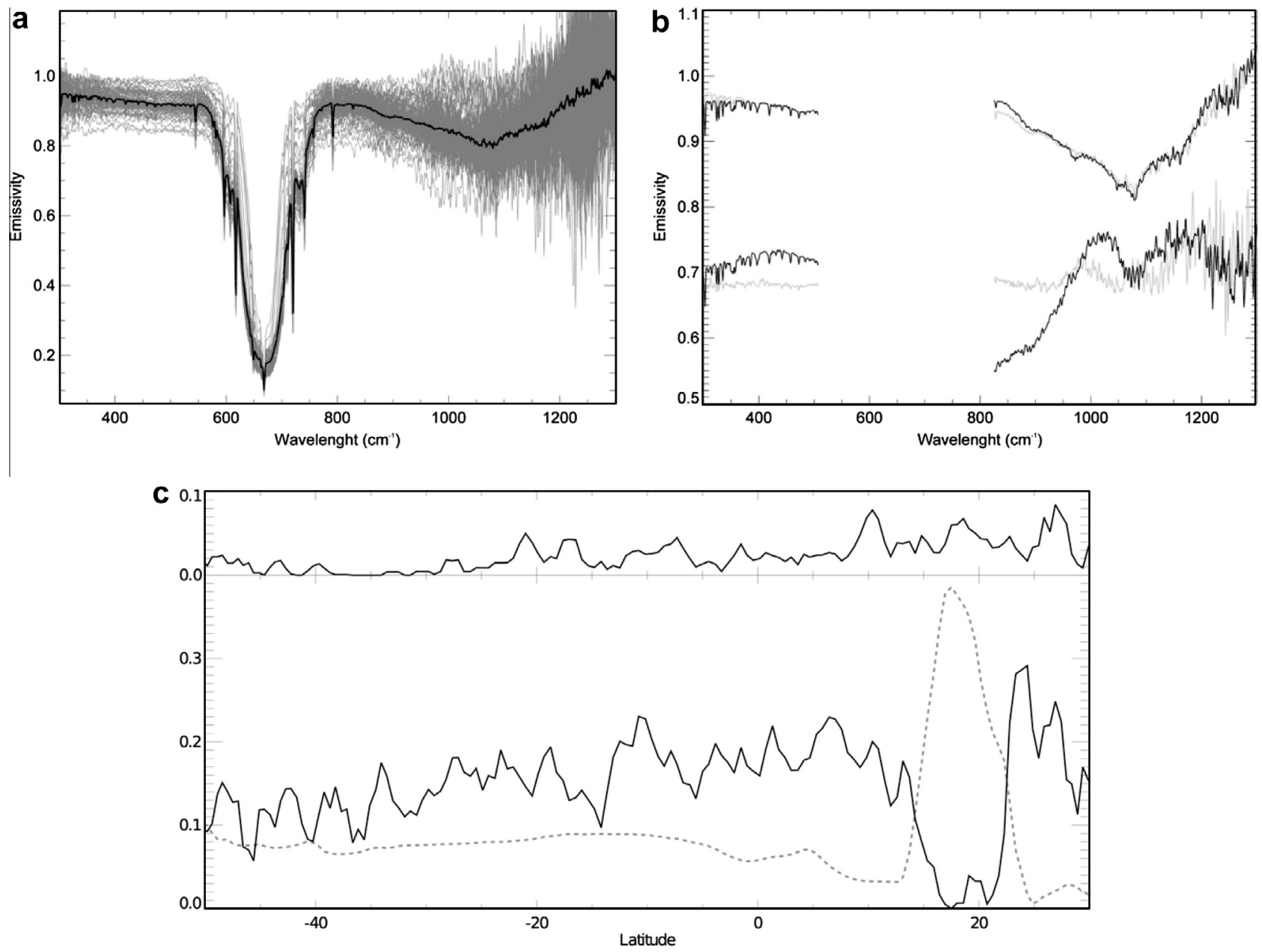

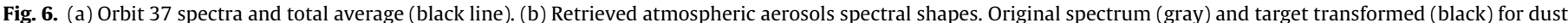

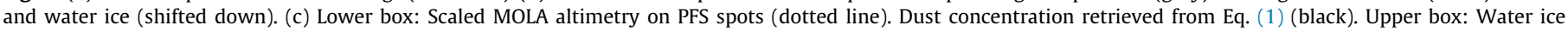
concentration. 


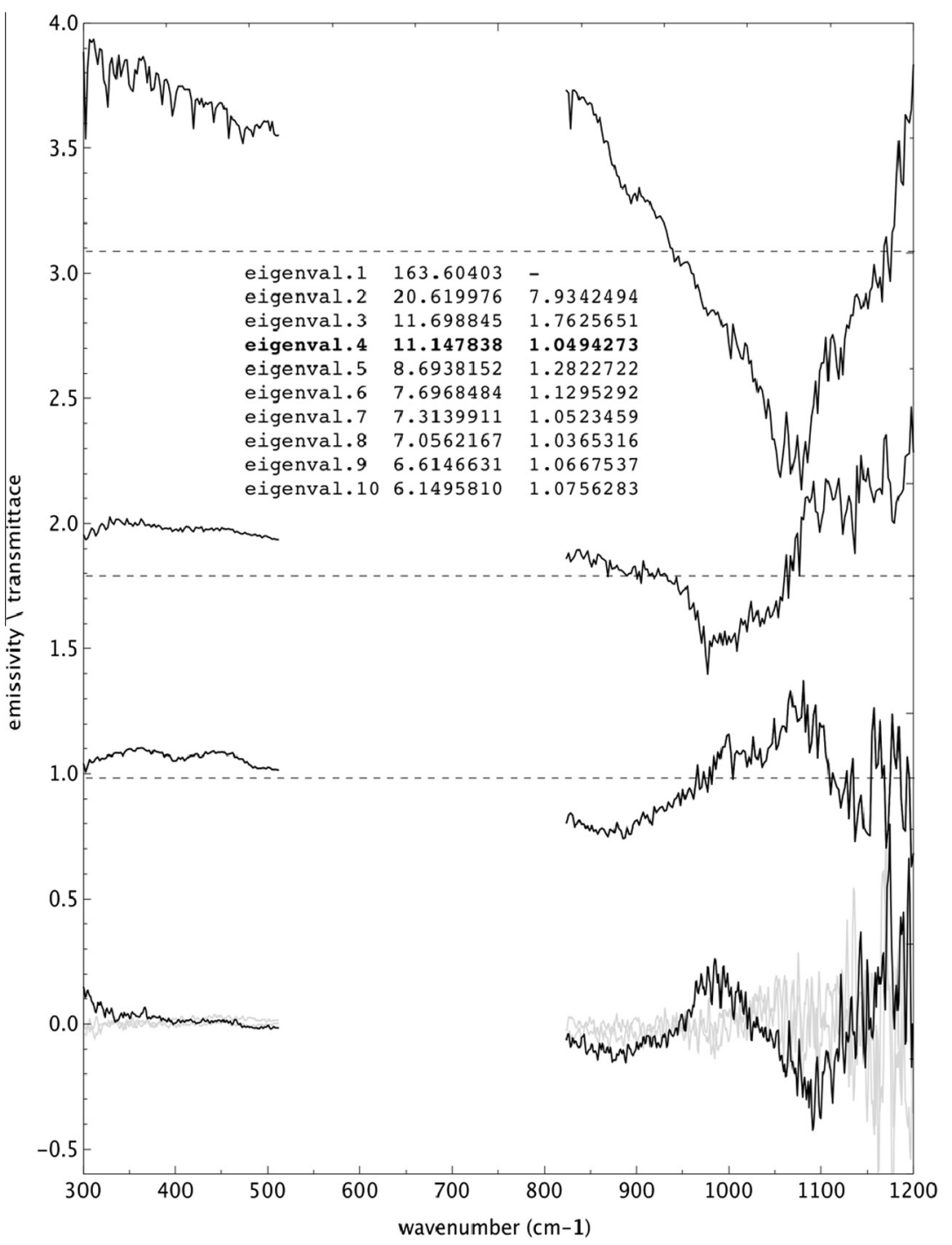

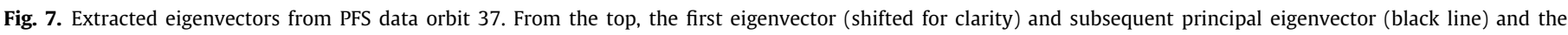
secondary eigenvector (gray line). The secondary are clearly related mostly to noise. The columns show the eigenvalues and their ratio.

variation in the water ice particles effective radius (Wolff, 2003) relative to the population represented by the general PFS atmospheric water ice cloud end-members. Moreover, and the general higher noise is coherent with lower abundance or variation of water ice in the atmosphere, even if two peaks on the volcano flanks could be related to high-altitude orographic clouds.

Whether the $1080 \mathrm{~cm}^{-1}$ band is or is not associated to the residual dust cannot be concluded from this analysis and a further investigation is needed to address this particular issue.

As in the previous application, no surface signature was found among the extracted eigenvectors. The successful separation of the atmospheric end-members allows the extraction of their relative abundance by manipulation of the Eq. (1). From the latitudinal profile in Fig. $6 \mathrm{c}$ the strong influence of the Olympus Mons caldera on the atmosphere through the variation of atmospheric endmembers abundance is evident.

\subsection{Comparison between TES, PFS and IRIS}

The 1-year investigation of the Mariner 9 IRIS instrument around Mars started on November 14th, 1971. The data collected by this instrument has been a great source of information, especially for the understanding of the martian atmosphere (Santee and Crisp, 1993; Grassi and Formisano, 2000; Formisano, 2001; Zasova, 2001).

It is of particular interest to compare its results with the ones from TES and PFS because it covers nearly the same spectral range (roughly $200-2000 \mathrm{~cm}^{-1}$ ). The spectral resolution of IRIS of $\sim 2.4 \mathrm{~cm}^{-1}$ is close to the $\sim 2 \mathrm{~cm}^{-1}$ LWC PFS spectral resolution (further details about IRIS in Hanel et al., 1972).

The IRIS data do not map Mars completely in terms of diurnal, seasonal, and latitudinal changes. Nevertheless, these data represent a unique opportunity to have a look at the recent past of the martian atmosphere. Hence, we analyzed the data collected by the IRIS instrument in order to better characterize the long-scale temporal variability of atmospheric constituents.

We filtered the whole IRIS data set of around 20,000 spectra from the Mariner 9 nominal mission phase. We selected only warm daily surfaces and, to emphasize atmospheric components, we focused on periods of relatively high dust opacity as well as using data collected over surfaces with high emissivity. These surfaces were selected based on previous TES results (Bandfield et al., 2000; Smith et al., 2000b). We obtained about 4400 spectra, spanning from Ls $\sim 290^{\circ}$ to $\sim 350^{\circ}$, essentially during the northern winter, and with a local time comprised between 9 and 17 .

In contrast with PFS data, we did not retrieve atmospheric opacity directly from the IRIS data. Thus we separated the spectra based 
on the average emissivity in small window of $25 \mathrm{~cm}^{-1}$ centered on the main atmospheric absorber bands. The most representative bands are at $850 \mathrm{~cm}^{-1}$ for the water ice cloud and at $1075 \mathrm{~cm}^{-1}$ for the mineral dust.

This procedure does not retrieve the atmospheric particulates total opacity, but these values could be used to select a set of representative spectra for varying opacity level in the selected window, as we did for the PFS data in Section 5.1, assuming that the observed emissivity in every window is affected essentially only by only one opaque absorber.

The analysis of principal factors (see Section 4.1) allows to extract the varying components in the dataset, that again are clearly linked to the dust and water ice cloud spectral shape.

Again, we translate these abstract results into physically meaningful spectra, by means of the target transformation, using the
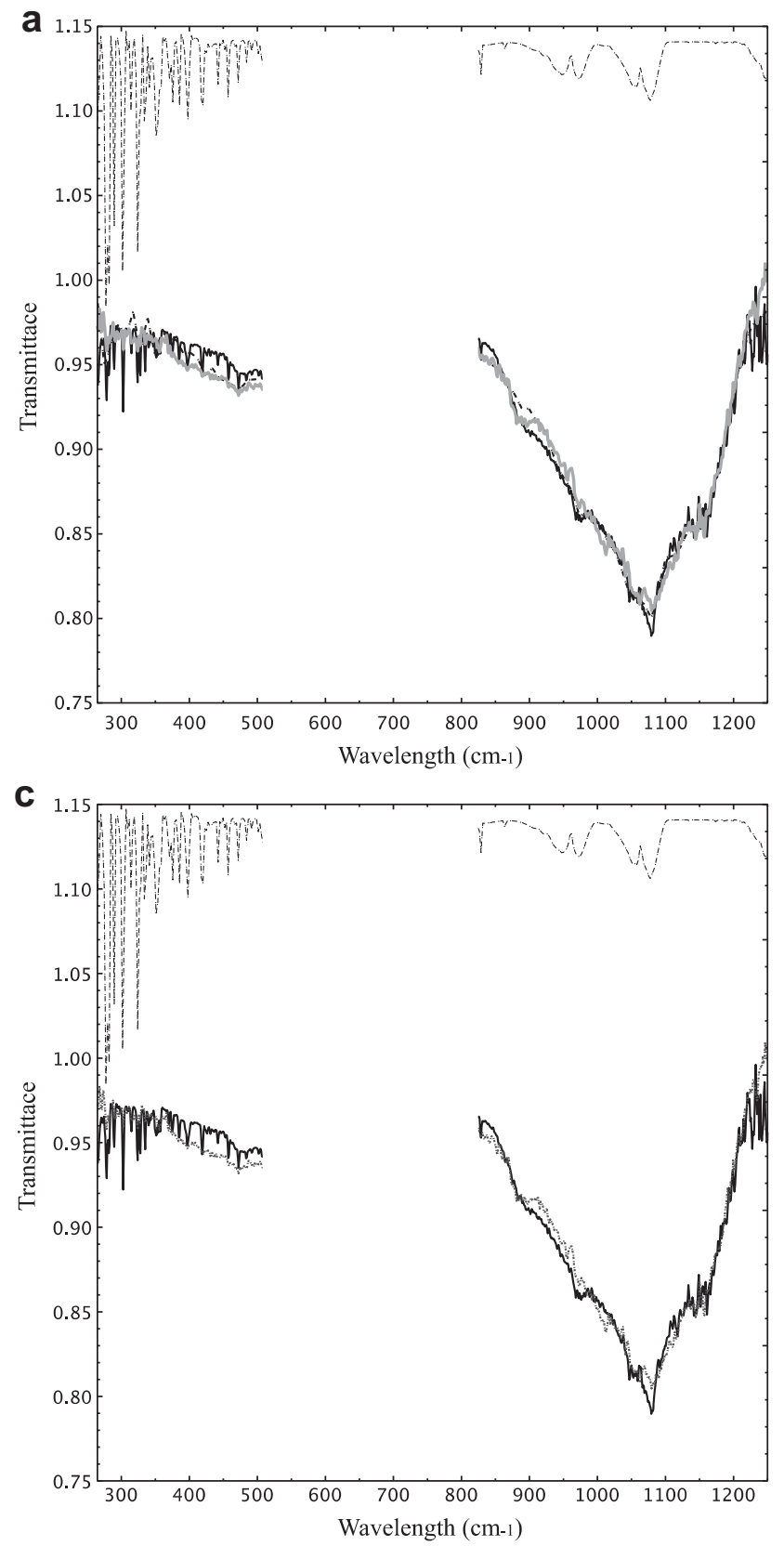

general atmospheric shape obtained from TES as an initial guess. The dust end-members resulting from the IRIS data analysis were directly compared to PFS and TES results. It is evident that dust spectral shapes in Fig. 8 largely coincide, indicating that the dust population described by the end-members we extracted from the data remains almost constant during the time monitored by the three instruments.

As seen with PFS, here the gaseous line could be clearly resolved in the spectral shape representing the dust.

It is worth mentioning that, as in the case of TES and PFS, the ice clouds component exhibits a greater noise, again due to a low concentration component that generally produces a nosier spectral shape resulting in a worse separation. Excluding some seasonal episodic events, in many case the ice clouds represent a weaker atmospheric component in the martian atmosphere.
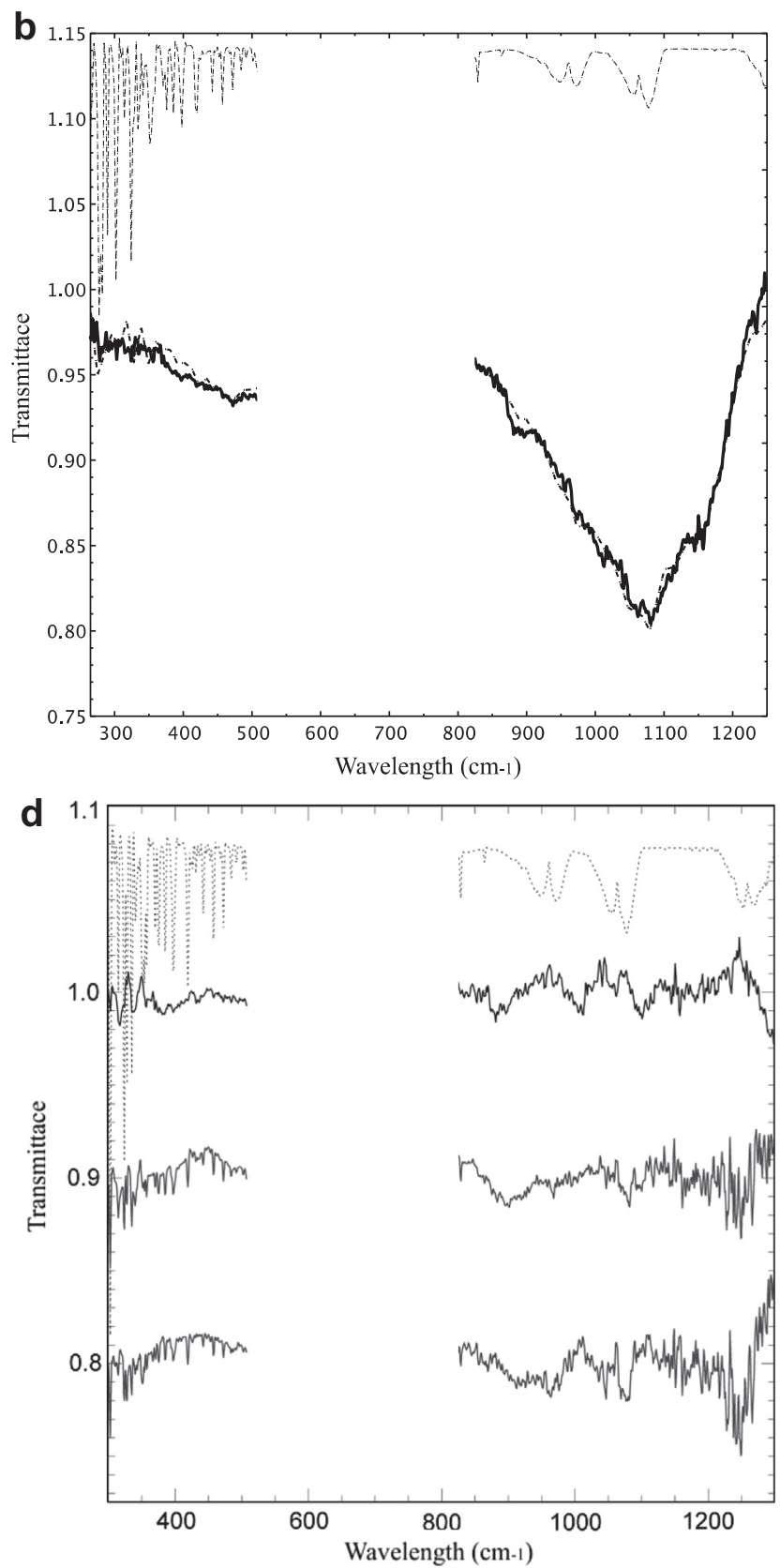

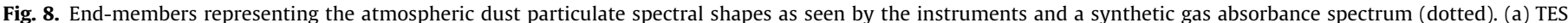

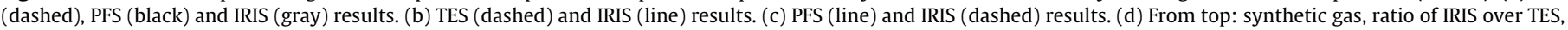
ratio of PFS over TES and ratio of IRIS over PFS. 
Therefore, these preliminary results for water ice need further improvements, most likely selecting a smaller dataset suited to specifically address ice cloud issues.

In the IRIS dust end-member the band at $1080 \mathrm{~cm}^{-1}$ is absent, reinforcing the idea that the band clearly present in the PFS endmember in Fig. 8 could be linked to the other effects, probably residual instrumental effects.

Then, the same considerations drawn for the general PFS endmembers are still valid and the structure bearing to gaseous lines and bands are clearly resolved, despite IRIS' slightly lower spectral resolution of $\sim 2.4 \mathrm{~cm}^{-1}$.

\section{Conclusions}

In the present work we successfully identified a component in the atmospheric dust that is extremely stable over almost 30 years of martian observations. There are no main variations in the spectral shapes representing this atmospheric dust component that can address physical changes in the particle sizes or in the composition of the atmospheric constituents themselves.

This means that there is a dust population in the overall mineral haze, whose transmission spectra observed at different time, do not reveal mineral structural differences linked to the presence of specific molecular groups nor change of Reststrahlen bands strength, which would indicate a change of particles' physical dimensions.

Considering also that the IRIS data gives information on the atmospheric component as it was during 1971, while the TES and PFS data observed the martian atmosphere almost continuously starting from 1997 to present, we believe that this is a further proof of the homogeneity of the planetary dust source.

Moreover, in this paper we present the first successful application to high-resolution data coming from the PFS instrument of the target transformation and factor analysis techniques, showing how the PFS data can be successfully modeled as a combination of the self-consistently recovered atmospheric end-members. The PFS higher spectral resolution offers the chance to better discriminate the contribution from the gaseous atmospheric components upon their narrow spectral signatures. The spectral end-members retrieved are subject of an ongoing study, to investigate the composition and seasonal evolution of atmospheric components.

Despite the caution employed in selecting data with little or no surface influence, we suspect that the PFS dust spectral shapes could still include a small surface contribution, since the PFS dust end-member ratio resembles surface emissivity spectra derived by TES observations for high wavenumbers. Further study on this specific issue coherently demonstrates that it is possible to efficaciously extract information about the surface and to analyze regions of the martian surface using PFS observations (Maturilli et al., 2009; Palomba et al., 2009).

\section{References}

Bandfield, J.L., Christensen, P.R., Smith, M.D., 2000. Spectral data set factor analysis and end-member recovery: Application to analysis of martian atmospheric particulates. J. Geophys. Res. 105, 9573-9587.

Benson, J.L., Bonev, B.P., James, P.B., Shan, K.J., Cantor, B.a., Caplinger, M.a., 2003. The seasonal behavior of water ice clouds in the Tharsis and Valles Marineris regions of Mars: Mars Orbiter Camera observations. Icarus 165, 34-52.

Cantor, B., 2002. Multiyear Mars Orbiter Camera (MOC) observations of repeated martian weather phenomena during the northern summer season. J. Geophys. Res. 107, 5014-5021.

Clancy, R.T., 2003. Mars aerosol studies with the MGS TES emission phase function observations: Optical depths, particle sizes, and ice cloud types versus latitude and solar longitude. J. Geophys. Res. 108, 5098-5117.

Clancy, R.T. et al., 2000. An intercomparison of ground-based millimeter, MGS TES and Viking atmospheric temperature measurements: Seasonal and interannual variability of temperatures and dust loading in the global Mars atmosphere. J. Geophys. Res. 105, 9553-9571.
Edgett, K.S., Malin, M.C., 2000. New views of Mars eolian activity, materials, and surface properties: Three vignettes from the Mars Global Surveyor Mars Orbiter Camera. J. Geophys. Res. 105, 1623-1650.

Farmer, V.C., 1974. The Infrared Spectra of Minerals. Mineralogical Society, London. Formisano, V., 2001. IRIS Mariner 9 data revisited: Water and dust daily cycles. Planet. Space Sci. 49, 1331-1346.

Formisano, V. et al., 2005. The Planetary Fourier Spectrometer (PFS) onboard the European Mars Express mission. Planet. Space Sci. 53, 963-974.

Gillespie, A.R., 1992. Spectral mixture analysis of multispectral thermal infrared images. Rem. Sens. Environ. 42, 137-145.

Giuranna, M. et al., 2005. Calibration of the Planetary Fourier Spectrometer long wavelength channel. Planet. Space Sci. 53, 993-1007.

Grassi, D., Formisano, V., 2000. IRIS Mariner 9 data revisited: 2. Aerosol dust composition. Planet. Space Sci. 48, 577-598.

Grassi, D. et al., 2005. Methods for the analysis of data from the Planetary Fourier Spectrometer on the Mars Express Mission. Planet. Space Sci. 53, 1017-1034.

Hamilton, J.C., Gemperline, P.J., 1990. Mixture analysis using factor analysis. II: Selfmodeling curve resolution. J. Chemomet. 4, 1-13.

Hamilton, V.E., McSween, H.Y. Hapke, B., 2005. Mineralogy of martian atmospheric dust inferred from thermal infrared spectra of aerosols. J. Geophys. Res. 110, 111.

Hanel, R.a. et al., 1972. Infrared spectroscopy experiment on the Mariner 9 mission: Preliminary results. Science (New York, NY) 175, 305-308.

Hapke, B., 1981. Bidirectional reflectance spectroscopy 1. Theory. J. Geophys. Res. 86, 3039-3054.

Hechler, M., Yáñez, A., 2003. Mars express orbit design. Acta Astronaut. 53, 497507.

Ignatiev, N.I., Grassi, D., Zasova, L.V., 2005. Planetary Fourier Spectrometer data analysis: Fast radiative transfer models. Planet. Space Sci. 53, 1035-1042.

Malinowski, E.R., 2002. Factor Analysis in Chemistry, third ed. New York, NY, USA.

Maturilli, A., Helbert, J., Mario, D., 2009. Phyllosilicates detection in Syrtis Major and Mawrth Vallis of Mars from PFS measured spectra. In: European Planetary Science Congress 2009, Potsdam, Germany, p. 106.

Moersch, J.E., Christensen, P.R., 1995. Thermal emission from particulate surfaces: A comparison of scattering models with measured spectra. J. Geophys. Res. 100, 7465-7477.

Montmessin, F., Bertaux, J.-L., Quémerais, E., Korablev, O., Rannou, P., Forget, F., Perrier, S., Fussen, D., Lebonnois, S., Rébérac, A., Dimarellis, E., 2006. Subvisible $\mathrm{CO}_{2}$ ice clouds detected in the mesosphere of Mars. Icarus 183, 403-410.

Palomba, E., Zinzi, A., Cloutis, E.A., D’Amore, M., Grassi, D., Maturilli, A., 2009. Evidence for Mg-rich carbonates on Mars from a $3.9 \mu \mathrm{m}$ absorption feature. Icarus 203, 58-65.

Pearl, J.C., Smith, M.D., Conrath, B.J., Bandfield, J.L., Christensen, P.R., 2001. Observations of martian ice clouds by the Mars Global Surveyor Thermal Emission Spectrometer: The first martian year. J. Geophys. Res. 106, 1232512338.

Ramsey, M.S., Christensen, P.R., 1998. Mineral abundance determination: Quantitative deconvolution of thermal emission spectra. J. Geophys. Res. 103, 577-596.

Ramsey, M.S., Howard, D.A., Christensen, P.R., Lancaster, N., 1993. Mineralogic variability of the Kelso dunes, Mojave desert, California derived from Thermal Infrared Multispectral Scanner (TIMS) data. In: Realmuto, V.J. (Ed.), Sum. of the Fourth Ann. Airborne Geosci. Workshop, vol. 2, TIMS, Workshop, pp. 9-12.

Salisbury, J.W., 1991. Infrared (2.1-25 $\mu \mathrm{m})$ Spectra of Minerals. John Hopkins University Press.

Salisbury, J.W., Hapke, B., Eastes, J.W., 1987. Usefulness of weak bands in midinfrared remote sensing of particulate planetary surfaces. J. Geophys. Res. 92, 702-710.

Santee, M., Crisp, D., 1993. Thermal structure and dust loading of the martian atmosphere during late southern summer: Mariner 9 revisited. J. Geophys. Res. 98, 3261-3279.

Smith, M.D., 2004. Interannual variability in TES atmospheric observations of Mars during 1999-2003. Icarus 167, 148-165.

Smith, M.O., Adams, J.B., Johnson, P.E., 1985. Quantitative determination of mineral types and abundances from reflectance spectra using principal components analysis. J. Geophys. Res. 90 (Suppl.), 797-804.

Smith, M.D., Bandfield, J.L., Christensen, P.R., 2000a. Separation of atmospheric and surface spectral features in Mars Global Surveyor Thermal Emission Spectrometer (TES) spectra. J. Geophys. Res. 105, 9589-9607.

Smith, M.D., Pearl, J.C., Conrath, B.J., Christensen, P.R., 2000b. Mars Global Surveyor Thermal Emission Spectrometer (TES) observations of dust opacity during aerobraking and science phasing. J. Geophys. Res. 105, 9539-9552.

Smith, M.D., Pearl, J.C., Conrath, B.J., Christensen, P.R., 2001. Thermal Emission Spectrometer results: Mars atmospheric thermal structure and aerosol distribution. J. Geophys. Res. 106, 23929-23945.

Thomson, J.L., Salisbury, J.W., 1993. The mid-infrared reflectance of mineral mixtures (7-14 $\mu \mathrm{m})$. Rem. Sens. Environ. 45, 1-13.

Wolff, M.J., 2003. Constraints on the size of martian aerosols from Thermal Emission Spectrometer observations. J. Geophys. Res. 108, 5097-5119.

Zasova, L., 2001. The martian atmosphere in the region of the great volcanoes: Mariner 9 IRIS data revisited. Planet. Space Sci. 49, 977-992.

Zasova, L. et al., 2005. Water clouds and dust aerosols observations with PFS MEX at Mars. Planet. Space Sci. 53, 1065-1077.

Zinzi, A., Palomba, E., Rinaldi, G., D’Amore, M., 2010. Effect of atmospheric dust loading on martian albedo measurements. Icarus 208, 590-597. 